\title{
A Version of Hilbert's 13th Problem for Infinitely Differentiable Functions
}

\author{
Shigeo Akashi and Satoshi Kodama \\ Department of Information Sciences, Faculty of Science and Technology, Tokyo University of Science, \\ 2641 Yamazaki, Noda City, Chiba Prefecture 278-8510, Japan \\ Correspondence should be addressed to Shigeo Akashi, akashi@is.noda.tus.ac.jp
}

Received 29 December 2009; Accepted 4 March 2010

Academic Editor: Anthony To Ming Lau

Copyright (C) 2010 S. Akashi and S. Kodama. This is an open access article distributed under the Creative Commons Attribution License, which permits unrestricted use, distribution, and reproduction in any medium, provided the original work is properly cited.

In 1957, Kolmogorov and Arnold gave a solution to the 13th problem which had been formulated by Hilbert in 1900. Actually, it is known that there exist many open problems which can be derived from the original problem. From the function-theoretic point of view, Hilbert's 13th problem can be exactly characterized as the superposition representability problem for continuous functions of several variables. In this paper, the solution to the superposition representability problem for infinitely differentiable functions of several variables is given.

\section{Superposition Representability and Superposition Irrepresentability}

In 1957, Kolmogorov and Arnold [1] solved Hilbert's 13th problem asking if all continuous real-valued functions of several real variables can be represented as superpositions of continuous functions of fewer variables. Moreover, in 1964, Vitushkin [2] solved the problem, which had been derived from Hilbert's 13th problem, asking if all finitely differentiable realvalued functions of several real variables can be represented as superpositions of finitely differentiable functions of fewer variables. In this paper, the solution to the superposition representability problem for infinitely differentiable functions of several variables is given.

Let $\mathcal{F}(D)_{3}$ (resp., $\mathcal{F}(D)_{2}$ ) be a set of functions of three variables (resp., two variables) satisfying the condition $P$ such as continuity or differentiability. Then, the superposition representability can be classified into the following two concepts.

Strong representabity: there exists a positive integer $k$ satisfying that, for any function $f$ of $\mp(D)_{3}, f$ can be represented as a $k$-time nested superposition constructed from $2^{k+1}-1$ functions of $\mathcal{F}(D)_{2}$. 
Weak representability: for any function $f$ of $\mathscr{F}(D)_{3}$, there exists a positive integer $k_{f}$ such that $f$ can be represented as a $k_{f}$-time nested superposition constructed from several functions of $\mathcal{F}(D)_{2}$.

Here, for a certain condition $D, D$ is said to be strongly (resp., weakly) representable, if strong (resp., weak) representability under the condition $P$ holds. It is clear that $D$ is weakly representable, if $D$ is strongly representable. By the same way as above, the superposition irrepresentability can be also classified into the following two concepts.

Strong irrepresentability: there exists a function $f$ of $\mathscr{f}(D)_{3}$ which cannot be represented as any finite-time nested superposition constructed from several functions of $\mathcal{F}(D)_{2}$.

Weak irrepresentability: for any positive integer $k$, there exists a function $f_{k}$ of $\mathcal{F}(D)_{3}$ which cannot be represented as any $k$-time nested superposition constructed from several functions of $\mathcal{F}(D)_{2}$.

Here, for a certain condition $D, D$ is said to be strongly (resp., weakly) irrepresentable, if strong (resp., weak) irrepresentability under the condition $D$ holds. It is clear that $D$ is weakly irrepresentable, if $D$ is strongly irrepresentable. Moreover, it is also clear that $D$ is weakly irrepresentable (resp., representable), if $p$ is not strongly representable (resp., irrepresentable). Therefore, we can classify a condition such as continuity or differentiability into three cases.

Case 1. The case that $D$ is strongly representable.

Case 2 . The case that $D$ is not only weakly representable but also weakly irrepresentable.

Case 3. The case that $D$ is strongly irrepresentable.

For example, if we take continuity as an example of $p$, then owing to Kolmogorov and Arnold, we can say that continuity satisfies Case 1 . As for the proof, we refer to [1, 3]. Moreover, if we take analyticity as an example of $\mathcal{D}$, then, owing to Babenko, Erohin, and Akashi, we can say that analyticity satisfies Case 3. As for the proof, we refer to [4-7]. If we take finite differentiability as an example of $D$, then, owing to Vituskin, we can say that finite differentiability satisfies Case 3. As for the proof, we refer to [2]. It is clear that polynomial condition satisfies Case 2. In the following section, this result will be formulated as a generalized relation between polynomial condition and infinite differentiability condition.

Recently, it is discussed that Hilbert's 13th problem can be applied to the theory of multidimensional numerical data compression. Since the results stated above show that it is important for any functions of three variables to find the appropriate superpositions which can approximate most efficiently to the original function. Therefore, nonlinear theoretic approximation methods will play important roles in the theory of multidimensional numerical data compression. As for the nonlinear theoretic approximation methods, we can refer to Takahashi's results [8].

\section{Weak Irrepresentability of Polynomial Condition}

In this section, we prove that polynomial condition is weakly irrepresentable.

Lemma 2.1. For any positive integer $n$, there exists a polynomial $f_{n}$ which cannot be represented as any n-time nested superposition constructed from several infinitely differentiable functions of two variables on $(-1,1)^{2}$. 
Proof. Let $i, j$, and $k$ be three nonnegative integers. Then, for any infinitely differentiable functions $f(\cdot, \cdot)$ and $g(\cdot, \cdot, \cdot)$, we define $c(f)_{i, j}$ and $c(g)_{i, j, k}$ as

$$
\begin{gathered}
c(f)_{i, j}=\left.\frac{\partial^{i+j} p}{\partial^{i} x \partial^{j} y}\right|_{x=0, y=0}, \\
c(g)_{i, j, k}=\left.\frac{\partial^{i+j+k} p}{\partial^{i} x \partial^{j} y \partial^{k} z}\right|_{x=0, y=0 . z=0},
\end{gathered}
$$

respectively. Here, assume that there exists a positive integer $\ell$ satisfying that all the polynomials can be represented as $\ell$-time nested superpositions. Then, the total number of infinitely differentiable functions of two variables, from which we use to construct the $\ell$ time nested superposition, is less than or equal to $2^{\ell}-1$. Let $p(\cdot, \cdot, \cdot)$ be a polynomial of three variables and $\left\{f_{p, m}(\cdot, \cdot) ; 1 \leq m \leq 2^{\ell}-1\right\}$ is a family of infinitely differentiable functions of two variables from which we use to construct the $\ell$-time nested superposition of $p$. For any positive integer $m$ which is less than or equal to $2^{\ell}-1$ and for any nonnegative integer $r$, we have

$$
\begin{aligned}
\operatorname{card}\left(\left\{c\left(f_{p, m}\right)_{i, j} ; i+j=r\right\}\right) & ={ }_{2} H_{r} \\
& =\frac{(r+1) !}{r ! 1 !} \\
& =r+1, \\
\operatorname{card}\left(\left\{c(p)_{i, j, k} ; i+j+k=r\right\}\right) & ={ }_{3} H_{r} \\
& =\frac{(r+2) !}{r ! 2 !} \\
& =\frac{(r+2)(r+1)}{2} .
\end{aligned}
$$

Since Taylor's expansion theorem assures that, for any nonnegative integer $r,\left\{c(p)_{i, j, k} ; 0 \leq\right.$ $i+j+k \leq r\}$ can be exactly characterized as $\bigcup_{m=1}^{2^{\ell}-1}\left\{c\left(f_{p, m}\right)_{i, j} ; 0 \leq i+j \leq r\right\}$, we have

$$
\operatorname{card}\left(\left\{c(p)_{i, j, k^{\prime}} ; 0 \leq i+j+k \leq r\right\}\right) \leq \sum_{m=1}^{2^{\ell}-1} \operatorname{card}\left(\left\{c\left(f_{p, m}\right)_{i, j^{\prime}} 0 \leq i+j \leq r\right\}\right) .
$$

This implies that, for any positive integer $s$, the following inequality holds:

$$
\sum_{r=0}^{s} \frac{(r+2)(r+1)}{2} \leq \sum_{r=0}^{s}\left(2^{\ell}-1\right)(r+1)
$$

So, we have a contradiction. 
Remark 2.2. This lemma shows that polynomial condition satisfies Case 2, because this condition is also weakly representable.

\section{Strong Irrepresentability of Infinite Differentiability Condition}

In this section, we prove that infinite differentiability condition is strongly irrepresentable.

Theorem 3.1. There exists an infinitely differentiable function defined on $(0,1)^{3}$ with values in $\mathbb{R}$, which cannot be represented as any superposition constructed from several infinitely differentiable functions defined on $(0,1)^{2}$ with values in $\mathbb{R}$.

Proof. Let $\phi(\cdot)$ be the function on $\mathbb{R}$ with values in $\mathbb{R}$ defined as

$$
\phi(x)= \begin{cases}0, & x \leq 0, \\ \exp \left[-\frac{1}{x}-\frac{1}{1-x}\right], & 0<x<1, \\ 0, & x \geq 1 .\end{cases}
$$

Then, it can be easily proved that $\phi$ is infinitely differentiable. Moreover, for any positive integer $n$, let $p_{n}(\cdot)$ and $q_{n}(\cdot)$ be the functions on $\mathbb{R}$ with values in $\mathbb{R}$ defined as

$$
\begin{gathered}
p_{n}(x)=(1-x)\left(1-\frac{1}{2^{n-1}}\right)+x\left(1-\frac{1}{2^{n}}\right) \\
q_{n}(x)=p_{n}^{-1}(x),
\end{gathered}
$$

respectively. For any positive integer $n$, Lemma 2.1 assures that there exists a polynomial $h_{n}(\cdot, \cdot, \cdot)$, which is defined on $(0,1)^{3}$ and cannot be represented as $n$-time nested superposition constructed from several polynomials of two variables. Therefore, it is sufficient that the following function $g(\cdot, \cdot, \cdot)$, which is defined as

$$
g(x, y, z)=\sum_{n=0}^{\infty} h_{n}\left(q_{n}(x), q_{n}(y), q_{n}(z)\right) \phi\left(q_{n}(x)\right) \phi\left(q_{n}(y)\right) \phi\left(q_{n}(z)\right), \quad 0<x, y, z<1,
$$

cannot be represented as any superposition constructed from several infinitely differentiable functions defined on $(0,1)^{2}$. Here, assume that, for a certain positive integer $k, g(\cdot, \cdot, \cdot)$ can be represented as $k$-time nested superposition. Then, for any positive integer $n, h_{n}(\cdot, \cdot, \cdot)$ is also characterized by the following equality:

$$
h_{n}(x, y, z)=\frac{g\left(p_{n}(x), p_{n}(y), p_{n}(z)\right)}{\phi(x) \phi(y) \phi(z)}, \quad 0<x, y, z<1
$$

Actually, this equality shows that, for any positive integer $n, h_{n}(\cdot, \cdot, \cdot)$ can be represented as a certain $k+2$-time nested superposition constructed from several infinitely differentiable functions defined on $(0,1)^{2}$. Therefore, we have a contradiction. 
Remark 3.2. Theorem 3.1 shows that infinite differentiability condition satisfies Case 3. For any real variables $x, y, z$, it is clear that $x y+y z+z x$ can be represented as the following two-time nested superposition:

$$
x y+y z+z x=(x+y) z+y z
$$

Actually, if we apply the same method as stated above to this polynomial, it can be proved that $x y+y z+z x$ cannot be represented as any one-time nested superposition.

\section{Acknowledgments}

The authors would like to express their hearty thanks to the referee who has given several pieces of suggestive advice to make the original manuscript more comprehensible, and they are also so thankful to Professor Anthony To-Ming Lau for having communicated several times with us in the course of reviewing procedure.

\section{References}

[1] A. N. Kolmogorov, "On the representation of continuous functions of many variables by superposition of continuous functions of one variable and addition," Doklady Akademii Nauk SSSR, vol. 114, pp. 953956, 1957 (Russian).

[2] A. G. Vitushkin, "Some properties of linear superpositions of smooth functions," Doklady Akademii Nauk SSSR, vol. 156, pp. 1003-1006, 1964.

[3] G. G. Lorentz, Approximation of Functions, Holt Rinehart \& Winston, New York, NY, USA, 1966.

[4] K. I. Babenko, "Best approximations to a class of analytic functions," Izvestiya Akademii Nauk SSSR. Seriya Matematicheskaya, vol. 22, pp. 631-640, 1958 (Russian).

[5] V. D. Erohin, "Asymptotic theory of the $\varepsilon$-entropy of analytic functions," Doklady Akademii Nauk SSSR, vol. 120, pp. 949-952, 1958 (Russian).

[6] S. Akashi, "A version of Hilbert's 13th problem for analytic functions," The Bulletin of the London Mathematical Society, vol. 35, no. 1, pp. 8-14, 2003.

[7] S. Akashi, "A version of Hilbert's 13th problem for entire functions," Taiwanese Journal of Mathematics, vol. 12, no. 6, pp. 1335-1345, 2008.

[8] W. Takahashi, "Iterative methods for approximation of fixed points and their applications," Journal of the Operations Research Society of Japan, vol. 43, no. 1, pp. 87-108, 2000. 